\title{
Dealing with vasomotor symptoms in patients with breast cancer, what options do we have?
}

\begin{abstract}
Vasomotor symptoms (VMS) affect a large number of women during perimenopause and postmenopause, significantly impacting their quality of life; furthermore, their duration is uncertain, and often long. Women with a history of breast cancer tend to have more severe and frequent vasomotor symptoms than the general population. Numerous studies have shown that vasomotor symptoms are the most frequent adverse events of adjuvant therapy, and that up to $20 \%$ of breast cancer patients consider discontinuing treatment because of them, despite its benefit in reducing recurrence. While hormone replacement therapy (MHT) is regularly used in healthy women for the treatment of VMS, it is contraindicated in patients with a history of breast cancer. Therefore, it is mandatory that different treatment approaches be offered to women for whom hormone therapy is contraindicated. As for treatment selection, there are a wide range of nonhormonal options, both pharmacological and nonpharmacological. There are very few clinical data on nonpharmacological interventions, and the role of alternative and complementary therapies remains controversial. Lifestyle modifications have a positive impact on women's general health status, in addition to helping with climacteric symptoms. As for complementary therapies, only cognitive-behavioral therapy and hypnosis are recommended. The literature review shows that pharmacological agents, i.e. serotonin-norepinephrine reuptake inhibitors (SNRIs), selective serotonin reuptake inhibitors (SSRIs), antihypertensives and anticonvulsants, decrease the intensity and frequency of VMS, proving a clinically significant improvement. However, some SSRIs and SNRIs are strong inhibitors of cytochrome P450 2D6 (CYP2D6), which affects the endoxifen level, and therefore should be avoided in patients treated with tamoxifen. A better therapeutic option for these patients is the use of citalopram and venlafaxine, although their consequences on breast cancer recurrence and survival are unknown. The treatment of VMS with antidepressants shows a lower efficacy than the treatment with estrogens, and there are few publications comparing both treatments. There is also a lack of data as to the optimal duration of these treatments. Two antiepileptic drugs, gabapentin and pregabalin, have also proved to be effective. Some comparative investigations are ongoing, and once we have their results we may identify the best option for managing menopausal symptoms in women who have had breast cancer.
\end{abstract}

Keywords: breast cancer, vasomotor symptoms, antihypertensives, antidepressants, antiepileptics, hot flashes - nonhormonal treatment - alternative therapies - isoflavone - cimicifuga racemosa
Volume 2 Issue 4 - 2018

\author{
Pilnik Susana,' Belardo María Alejandra, ${ }^{2}$ \\ Cavanna Malena, ${ }^{3}$ De Nardo Bárbara, ${ }^{3}$ \\ Starvaggi Agustina, ${ }^{3}$ Gelin Marina ${ }^{3}$ \\ 'Gynecology Department of the Hospital, Gynecological \\ Endocrinology and Climacteric Section, Argentina \\ 2Department of the Hospital Italiano de Buenos Aires, \\ Gynecology Assistant Professor at the Instituto Universitario \\ del Hospital Italiano de Buenos Aires, Argentina \\ ${ }^{3}$ Department of the Hospital Italiano de Buenos Aires, \\ Gynecological Endocrinology and Climacteric Section Associate, \\ Argentina
}

Correspondence: Pilnik Susana, Department of Gynecology, Italiano de Buenos Aires Hospital,Argentina,Tel +54 | |47866549, Email susana.pilnik@hospitalitaliano.org.ar

Received: June 19,2018 | Published: August 13,2018

\section{Introduction}

Breast cancer is the most common type of cancer in the western world, and the second-leading cause of cancer death in women, affecting one out of nine women aged 85 years old in developed countries. ${ }^{1}$ In the United States alone, it is estimated that this year there will be a total of 40,610 breast cancer deaths. ${ }^{2}$ In Argentina it is estimated that there will be more than 19,000 new cases per year and due to early diagnosis and treatments nearly $70 \%$ will survive. This brings with it the need to have different therapeutic alternatives to palliate climacteric syndrome. ${ }^{3}$ Vasomotor symptoms (VMS) affect 40 to $80 \%$ of women during perimenopause and postmenopause, respectively, and most of them report symptoms as being of moderate to severe intensity. ${ }^{4}$ In general, out of 4 women, 1 is asymptomatic, 2 present moderate hot flashes, and 1 experiences severe hot flashes. Their peak intensity and frequency occur during the first 2 years of postmenopause. The North American Menopause Society (NAMS) $)^{5}$ describes hot flashes as a feeling of intense heat, with face and neck flushing, accompanied by perspiration and tachycardia, often followed by chills. ${ }^{6}$ Their intensity and frequency vary from case to case. As for their duration, results vary among the different studies. SWAN study (an observational study of the menopause transition), which enrolled 3302 women and had a 17-year follow up, showed an average total mean duration of 7.4 years and a mean persistence after the final menstrual period (FMP) of 4.5 years. The risk factors associated with a longer duration of hot flashes identified in the study included young women with a lower educational level, as well as stress, depression and anxiety. ${ }^{4}$ Other studies, including the Melbourne Women's.

Midlife Health Project, ${ }^{4}$ found a total duration of 5.2 years; and the Penn Ovarian Aging Study ${ }^{4}$ showed a total duration of 8.8 to 10.2 years and a post-FMP duration of 4.6 years. Furthermore, it is well known that the best independent predictor for total duration and post-FMP duration is the onset of symptoms in the early stages of the menopause transition. ${ }^{4}$ Women with breast cancer often 
experience menopause earlier than the average population as a result of the treatment they undergo. As most cancers are hormone sensitive, women receive systemic hormone therapy to prevent recurrence and mortality. Both tamoxifen and aromatase inhibitors are standard of care in these patients after surgical treatment. ${ }^{7}$ The use of antiestrogens, in addition to exacerbating symptoms of depression and anxiety, causes hot flashes in $60 \%-70 \%$ of patients. ${ }^{8}$ Carpenter et al.,${ }^{9}$ found that VMS are more common and severe in patients with a history of breast cancer as compared to healthy women (65\% and $16 \%$, respectively). In a case-controlled study, Harris et al., ${ }^{10}$ report that breast cancer survivors have 5.3 times more menopausal symptoms than controls, and use more alternative treatments. A survey carried out with 190 breast cancer survivors ${ }^{10}$ revealed that the most common complaints included VMS (65\%), night sweats (44\%), vaginal dryness $(44 \%)$, sleep insufficiency (44\%) and dyspareunia (26\%). ${ }^{11}$ Numerous studies $^{11,12}$ have shown that VMS are the most frequent adverse events of adjuvant hormone therapy, and that up to $20 \%$ of patients consider discontinuing treatment because of them, despite its benefit in reducing recurrence. Menopausal symptoms have a negative impact on quality of life. The search for therapeutic alternatives has been an important area of study over the past two decades, with the aim of finding effective non-estrogen therapies to help this women. ${ }^{13}$

\section{Nonhormonal treatment}

\section{Nonpharmacological approaches}

Gynecologists, as primary care physicians, play a critical role in detecting habits and behaviors that pose a risk to health. Treating VMS represents only part of the gynecological consult, which is a unique opportunity to drive lifestyle modifications, and to receive counseling on healthy habits and stopping harmful behaviors (such as providing smoking cessation counseling). As for the position of the main organizations, in 2015, the NAMS ${ }^{14}$ produced recommendations dividing interventions for treating hot flashes into three groups, by level of evidence:

I. Recommended interventions include cognitive-behavioral therapy and hypnosis.

II. Interventions recommended with caution because studies are inconclusive as to their impact on VMS include weight loss, mindfulness therapy, isoflavones and derivatives, and stellate ganglion block.

Interventions with inconclusive or insufficient evidence include exercising (aerobic exercise) and yoga, acupuncture, relaxation and cooling techniques (clothing adjustments, cool environments) and avoidance of triggers (alcohol, spicy foods). Additionally, in 2016, the International Menopause Society (IMS) ${ }^{15}$ produced recommendations as to lifestyle, dieting and exercise, categorizing them by level of evidence:

\section{Level of evidence [A]:}

a. Smoking should be avoided.

\section{Level of evidence $[\mathrm{B}]$ :}

I. Regular exercise is advised to reduce cardiovascular and total mortality.

II. Optimal exercise recommendation is at least 150 minutes of moderate-intensity exercise per week. Two additional weekly sessions of resistance exercise may provide further benefit.
III. Weight loss of only $5-10 \%$ is sufficient to improve many of the abnormalities associated with the insulin resistance syndrome

IV. The recommended intensity of aerobic activity should take into account the older adult's aerobic fitness.

V. The basic components of a healthy diet are: several servings/day of fruits and vegetables, whole grain fibers, fish twice a week and low total fat (the use of olive oil is recommended). Consumption of salt should be limited and the daily amount of alcohol should not exceed $30 \mathrm{~g}$ for men and $20 \mathrm{~g}$ for women.

VI. Lifestyle modifications include socializing, and being physically and mentally active.

\section{Alternative therapies}

It is important to know the mechanism of action and efficacy of every one of these options, as well as their contraindications, adverse effects and drug interactions, so that the physician may identify the best treatment for the woman in need, and offer her the best therapeutic option available. With regard to oncological diseases, their lower incidence in China and Japan compared to the US was attributed to a higher intake of soy in the Asian countries. Some studies have shown the potential benefit of isoflavones (phytoestrogen) for the prevention of colon, endometrial and ovarian cancer. On the other hand, in terms of breast cancer, studies have provided controversial results. Prepuberal exposition of breast acini to phytoestrogens may cause them to mature early and, therefore, offer protection. In contrast, postpuberal exposition without breast maturity may potentially increase the risk of cancer through their estrogen agonist action. In turn, it was suggested that genistein appears to have a proliferative action on in vivo breast epithelium. Consequently, the use of isoflavones is currently not recommended in women with a history of breast cancer. ${ }^{16}$ Cimicifuga Racemosa's (actaea racemosa, black cohosh, black bugbane, black snakeroot, fairy candle) active metabolite is unknown and its mechanism of action is unclear. ${ }^{14-17}$ It was initially believed that cimicifuga produced an estrogenic effect; however, current studies show that it seems to act as a partial agonist of serotonin receptors and opioid receptors, and that it seems to have a dopaminergic effect as well. ${ }^{18}$ No drug interactions are known, although it may interfere with tamoxifen; ${ }^{19}$ however, since its mechanism of action is unknown, its concomitant use with hormone therapies is not recommended in women with a personal history of hormone-dependent cancers. The role of these treatments remains controversial. Studies and metaanalyses have not consistently supported their efficacy, therefore more controlled clinical trials are needed..$^{20,21}$

\section{Pharmacological approaches}

On the other hand, pharmacological agents, including, but not limited to, clonidine, selective serotonin reuptake inhibitors (SSRIs), serotonin-norepinephrine reuptake inhibitors (SNRIs), gabapentin and pregabalin, are a nonhormonal alternative for this group of patients. Clonidine, a central action alpha 2-adrenergic agonist, has shown, approximately, a $46 \%$ mild to moderate efficacy in the reduction of hot flashes when taken orally or transdermally. In tamoxifen users, the $0.1 \mathrm{mg}$ dose, administered through both routes, has shown to reduce the frequency and severity of hot flashes, ${ }^{22}$ as well as sleep disorders; although it is less effective that venlafaxine, ${ }^{23}$ it causes fewer adverse effects. A 10 -study meta-analysis showed that clonidine is slightly more efficient than placebo in reducing vasomotor symptoms. Its use has been associated with several significant adverse 
effects, such as dry mouth, dizziness, hypotension, constipation and sedation, therefore its current clinical use has been limited. ${ }^{24}$ SSRIs and SNRIs are groups of drugs that have been widely studied for the treatment of VMS in patients who have had breast cancer. Although their mechanism of action is unclear, their benefit on VMS appears independently of their antidepressant effect and much earlier than with other therapies. ${ }^{25}$ They have proved to reduce hot flashes in up to $50 \%$ of cases, with a similar efficacy among tamoxifen users and nonusers. ${ }^{26,27}$ Between 10 and $20 \%$ of patients inform some adverse effects, such as headaches, nausea, loss of appetite, gastrointestinal intolerance, dry mouth, anxiety, agitation, sleep disorders and sexual dysfunction. These adverse events occur less frequently at low doses. In spite of these potential unwanted effects, SSRIs and SNRIs are an excellent alternative.

Depression and mood alterations are common after a breast cancer diagnosis. The use of a low dose of these pharmacological agents may notably improve the quality of life for these women. ${ }^{13}$ In a doubleblind, placebo-controlled, randomized trial ${ }^{28}$ with venlafaxine, an SNRI, in increasing doses of $37.5,75$, and $150 \mathrm{mg} /$ day in patients with breast cancer who took tamoxifen, it was observed that, after four weeks, hot flashes scores had a rapid and significant $37 \%$ and $61 \%$ decrease, depending on dose. The adverse effects were associated with a higher dose, and there was no drug interaction in the metabolism of tamoxifen. ${ }^{29}$ Both tamoxifen-induced depression and quality of life improved with the use of venlafaxine. ${ }^{30,31}$ Our work group $^{32}$ of the Climacteric Section of the Hospital Italiano has carried out an experience with $37.5 \mathrm{mg}$ of venlafaxine in 30 postmenopausal women with moderate to severe hot flashes, including breast cancer patients. The result was statistically significant, with a $66 \%$ reduction in hot flashes scores. Venlafaxine has been the most studied drug. A review of VMS treatments from 1966 to 2003 concludes that venlafaxine is an effective nonhormonal alternative for the relief of symptoms that could not have been managed with other therapies. ${ }^{33}$ Desvenlafaxine, the most active metabolite of venlafaxine, at 100mg doses has shown a rapid onset of action, efficacy and to be well tolerated, with a significant reduction of VMS of $64 \% .{ }^{34}$ At $50-\mathrm{mg}$ doses, fewer adverse events and lower discontinuation rates were observed. A later trial using two intermediate doses of 100 and 150 $\mathrm{mg}$ daily versus placebo found similar results, with a $67 \%$ reduction in the number of VMS and a similar toxicity profile ${ }^{35}$ Side effects, such as nausea, insomnia and dry mouth, were only noted in the first week of treatment. Some studies have shown a small but significant increase in blood pressure. ${ }^{36,37}$ However, Speroff et al. ${ }^{26}$ did not find a difference in adverse effects with placebo after 52 weeks of treatment.

Only when SSRIs were used there was a benefit in the concomitant depression that these patients commonly have, ${ }^{38}$ although the possibility of withdrawal syndrome occurring with the sudden suppression of treatment must be taken into account, mainly with short-acting agents, including desvenlafaxine, venlafaxine and paroxetine. In breast cancer patients, ${ }^{39}$ fluoxetine, an SSRI, has shown a 50\% reduction in hot flashes. Other investigators have obtained lower results; with a $36 \%$ reduction in frequency and a $46 \%$ reduction in intensity of hot flashes. ${ }^{40}$ The most common side effects include nausea, fatigue, insomnia, nervousness and constipation. Variable and contradictory results have been observed with the use of sertraline, another SSRI, in healthy postmenopausal women, both at 50-mg and 100-mg doses. ${ }^{41}$ However, in tamoxifen users, sertraline has shown to be superior to placebo in reducing VMS without altering their quality of life. Kimmik et al., ${ }^{42}$ found a $50 \%$ reduction in the frequency of hot flashes in 62 tamoxifen users with severe hot flashes. ${ }^{43}$ The International Menopause Society (IMS) does not recommend the use of fluoxetine or sertraline due to the fact that no significant reduction in vasomotor symptoms was found in placebo-controlled studies. ${ }^{15}$ In 2013, the FDA approved the use of paroxetine at 7.5-mg doses as treatment for moderate-severe hot flashes, constituting the only psychoactive drug approved for this purpose. The efficacy of paroxetine was established in two randomized, double-blind, placebo-controlled, multicenter clinical trials. Among a total of 1184 menopausal women who had a median of 10 moderate-to-severe hot flushes per day, paroxetine $7.5 \mathrm{mg}$ was shown to significantly decreases both the frequency and intensity of hot flashes. ${ }^{44}$

Another study evaluated the efficacy of paroxetine in the treatment of hot flushes and sleep disorders in women survivors of gynecological cancer, demonstrating a reduction of $40 \%-67 \%$ in the frequency of hot flushes, in women with a history of breast cancer. The benefits were observed in 1-2 weeks of treatment, persisting for 6 weeks. Regarding to sleep, paroxetine $7.5 \mathrm{mg}$ once a day significantly reduced the number of night awakenings attributed to VMS and increased sleep duration. Its effect occurred without increasing sedation, acting selectively on sleep parameters related to VMS. ${ }^{45,46}$ Given that we are suggesting the use of SSRIs and SNRIs in women with a history of breast cancer, it is important to consider drug interactions. Some drugs may interfere with the transformation of tamoxifen into its active metabolite, 4-hydroxy-desmethyl-tamoxifen (endoxifen), by inhibiting cytochrome P450 2D6 (CYP2D6) ${ }^{47}$ The US Food and Drug Administration (FDA) classifies these psychiatric drugs into strong, moderate and weak inhibitors, according to their interaction with cytochrome P450. Both paroxetine and fluoxetine have been classified as strong inhibitors; sertraline as a moderate inhibitor; and citalopram, escitalopram, venlafaxine and desvenlafaxine as weak inhibitors. ${ }^{7}$ There is evidence that paroxetine, with a more intense effect, followed by fluoxetine and sertraline, inhibits CYP2D6, mitigating the endoxifen production. Interactions between CYP2D6 polymorphisms and concomitant antidepressants may be associated with an altered tamoxifen activity. ${ }^{47}$ Kelly et al., ${ }^{48}$ found that the irreversible inhibition of CYP2D6 by paroxetine may reduce or abolish the survival advantage offered by tamoxifen treatment. This has not been seen with other antidepressants. The use of paroxetine concomitantly with tamoxifen is associated with an increased risk of death from breast cancer, which is linked to treatment duration. ${ }^{49}$ There is an estimation of one additional breast cancer death within five years for every 20 women treated. However, Haque et al, using the records of two California health plans, did not observe an increased risk of death in women who used tamoxifen and antidepressants, including paroxetine. $^{50}$

Whether the interference of SSRIs/SNRIs with CYP2D6 has any impact on breast cancer recurrence and survival is controversial; however, venlafaxine and citalopram should be considered as the best options for the treatment of vasomotor symptoms in these patients. ${ }^{48}$ The American Clinical Oncology Society recommends avoiding the use of antidepressants classified as strong inhibitors. ${ }^{7}$ The efficacy of citalopram, an SSRI with weak effect on noradrenaline, dopamine and GABA reuptake, in reducing hot flashes was compared to MHT, finding a higher efficacy with the use of citalopram. ${ }^{51}$ Citalopram achieved a reduction of VMS in cases where the response to treatment with venlafaxine had been insufficient. ${ }^{52}$ It has shown a $49-55 \%$ improvement in hot flashes ${ }^{53}$ however, its long-term efficacy has not been proven. At nine months, neither citalopram nor fluoxetine 
were better than placebo for the treatment of VMS. ${ }^{54}$ Escitalopram, an SSRI with weak effect on noradrenaline and dopamine reuptake, at increasing doses has shown a 55\% reduction in hot flashes in menopausal women or women in the menopause transition, with $4 \%$ of side effects and a $70 \%$ satisfaction rate. ${ }^{55}$ Escitalopram, at 10 - to $20-\mathrm{mg} /$ day doses, had a $50 \%$ reduction in frequency and severity of VMS in menopausal women. ${ }^{56}$ Symptoms reappeared quickly after treatment discontinuation.

Our experience in the Climacteric Section of the Hospital Italiano de Buenos Aires with the use of escitalopram at 5-mg and 10-mg $\operatorname{doses}^{57}$ in patients with severe hot flashes, a history of breast cancer, or with a contraindication for MHT, was similar or even superior to the percentages of most published studies. Our results have indicated a $60 \%$ reduction by week 1 , which is progressively maintained after one year of use, with good compliance and few side effects. An analysis of three randomized clinical trials was performed, including a study by the Fred Hutchinson Cancer Research Center, which enrolled 899 perimenopausal and postmenopausal women with moderate vasomotor symptoms. Interventions included escitalopram 10-20 $\mathrm{mg}$, low oral doses of 17-beta-estradiol, and venlafaxine $75 \mathrm{mg}$. The reduction in frequency of vasomotor symptoms was comparable and modest. ${ }^{58}$ In 2015, the North American Menopause Society (NAMS) ${ }^{14}$ published their position statement on the nonhormonal management of vasomotor symptoms associated with menopause. 340 original investigation articles and 105 systematic reviews were assessed. The NAMS panel concluded that the recommended therapies for menopausal and postmenopausal hot flashes are paroxetine 7.5-10$25 \mathrm{mg} /$ day; escitalopram 10-20 mg/day; citalopram 10-20 mg/day; desvenlafaxine 50-150 mg/day; and venlafaxine XR $37.5-150 \mathrm{mg} /$ day. Furthermore, treatment must begin with the lowest available dose and increase as necessary. No difference in efficacy on VMS was found when comparing venlafaxine, desvenlafaxine, paroxetine, citalopram and escitalopram..$^{59}$

Several research protocols are ongoing, one of them a multicenter study of women with breast cancer and women with a high risk of breast cancer who are receiving tamoxifen together with venlafaxine, citalopram, escitalopram, gabapentin or sertraline; its purpose is to evaluate blood levels of tamoxifen and endoxifen. It is estimated to end in May 2018. ${ }^{60}$ The efficacy of treating vasomotor symptoms with antidepressants is lower than the efficacy achieved with estrogens. However, few publications compare both treatments. There is no optimal treatment duration established, and it is recommendable that the dose be gradually reduced in the event of discontinuing treatment. Withdrawal syndrome, although not frequent at the doses prescribed for hot flashes, is the main problem with short half-life agents, such as venlafaxine and paroxetine. There are few studies designed for determining the frequency of these symptoms. ${ }^{15-61}$ Gabapentin, a gamma-aminobutyric acid, regularly used as treatment for epilepsy and chronic neuropathic pain, has shown to be efficient in reducing hot flashes in breast cancer patients. ${ }^{62}$ It has no drug interactions, does not cause sexual dysfunction and seems to be well tolerated. The recommended dose for the treatment of hot flashes is $900 \mathrm{mg} /$ day ${ }^{63}$ As for adverse events, about $20 \%$ of patients report drowsiness, dizziness and some anhedonia, which appear following the first week of treatment and resolve without treatment by week four of use. ${ }^{64}$

Three meta-analyses have shown it to be superior to placebo. ${ }^{13}$ Some breast cancer patients develop neuropathic pain; gabapentin may help not only with the treatment of VMS, but also with the relief of pain. It is also an option for those who suffer from migraines. ${ }^{13}$ When comparing gabapentin with venlafaxine and MHT, high doses of gabapentin $\left(900 \mathrm{mg} /\right.$ day) were needed to reduce VMS. ${ }^{65}$ These doses were associated with more side effects. At 300-mg/day doses, a $55 \%$ improvement in VMS is seen, and at $900-\mathrm{mg}$ /day doses, $80 \%$ reductions are seen at 3 and 6 months. Due to their sedative effect, it is considered that a single night dose may be useful in patients who experience VMS accompanied by night sweats and repeated awakenings. ${ }^{66,67}$ Pregabalin is a gamma-aminobutyric acid (GABA) analogue with the same indications as gabapentin. A randomized, double-blind, placebo-controlled study comparing $75 \mathrm{mg}$ twice daily or $150 \mathrm{mg}$ twice daily of pregabalin and placebo found, after 6 weeks, a $65 \%$ and $71 \%$ improvement in the reduction of hot flashes with pregabalin, depending on dose. As for adverse effects, insomnia, dizziness and weight gain were observed; it is important to note that some women experienced cognitive dysfunction at higher doses. ${ }^{68}$ The Committee for the Evolution of Oncology Practices (CEPO), in a review of the literature published between 2000 and 2011, concluded that serotonin-norepinephrine reuptake inhibitors, selective serotonin reuptake inhibitors, antihypertensives and anticonvulsants significantly reduced the frequency and severity of hot flashes in breast cancer patients. ${ }^{69}$

\section{Conclusion}

As for nonpharmacological approaches to nonhormonal treatment of climacteric symptoms, randomized and controlled clinical trials with a higher number of patients are needed, so that scientific evidence of a higher quality may be achieved. The literature review shows that pharmacological agents, i.e. serotonin-norepinephrine reuptake inhibitors, selective serotonin reuptake inhibitors, and anticonvulsants, decrease the intensity and frequency of vasomotor symptoms, exhibiting a clinically significant improvement. Future studies will be necessary in order to identify the best management of menopausal symptoms in women who have had breast cancer and whose quality of life has been affected.

\section{Acknowledgements}

None.

\section{Conflict of interest}

The author declares there is no conflict of interest.

\section{References}

1. Bordeleau L, Pritchard K, Goodwin P, et al. Therapeutic options for the management of hot flushes in breast cancer survivors. An Evidence-Based Review. Clin Ther. 2007;29(2):230-241.

2. Cancer Facts \& Figures 2017. Atlanta American Cancer Society; 2017.

3. National Cancer Institute, Ministry of Health Argentina; 2016.

4. Avis NE, Crawford SL, Greendale G, et al. Duration of Menopausal Vasomotor Symptoms Over the Menopause Transition. JAMA Intern Med. 2015;175(4):531-539.

5. NAMS Website.

6. Deecher DC, Dorries K. Understanding the pathophysiology of vasomotor symptoms (hot flushes and night sweats) that occur in perimenopause, menopause, and postmenopause life stages. Arch Women's Mental Health. 2007;10(6):247-257. 
7. Irarrázaval M, Gaete L, Antidepressants agents in breast cancer patients using tamoxifen: review of basic and clinical evidence. Rev Med Chile. 2016;144(10):1326-1335.

8. Del Re M, Citi V, Crucitta S, et al, Pharmacogenetics of CYP2D6 and tamoxifen therapy: Light at the end of the tunnel? Pharmacol Res. 2016;107:398-406

9. Carpenter JS, Johnson D, Wagner L, Andrykowski M, Hot flashes and related outcomes in breast cancer survivors and matched comparison women. Oncol Nurs Forum. 2002;29(3):E16-E25.

10. Harris PF, Remington PL, Trentham-Dietz A, et al. Prevalence and treatment of menopausal symptoms among breast cancer survivors. J Pain Sympton Manage. 2002;23(6):501-509.

11. Mortimer JE. Hormone replacement therapy and beyond. The clinical challenge of menopausal symptoms in breast cancer survivors. Geriatrics. 2002;57:25-31.

12. Cella D, Fallowfield LJ. Recognition and management of treatmentrelated side effects for breast cancer patients receiving adjuvant endocrine therapy. Breast Cancer Res Treat. 2008;107(2):167-180.

13. Eden J. ENDOCRINE DILEMMA: Managing menopausal symptoms after breast cancer. Eur J Endocrinol. 2016;174(3):71-77.

14. Nonhormonal management of menopause-associated vasomotor symptoms: 2015 position statement of The North American Menopause Society. Menopause. 2015;22(11):1155-1174.

15. Baber RJ, Panay N, Fenton A, et al. 2016 IMS Recommendations on women's midlife health and menopause hormone therapy. Climateric. 2016;19(2):109-150.

16. Sirotkin A, Harrath A. Phytoestrogens and their effects. Eur J Pharmacol. 2014;74:230-236

17. Mintziori G, Lambrinoudaki I, Goulis DG, et al. EMAS position statement: Duration of Menopausal Vasomotor Symptoms Over the Menopause Transition. Maturitas. 2015;81(3):410-413.

18. Borrelli F, Ernst E. Alternative and complementary therapies for the menopause. Maturitas. 2010;66(4):333-343.

19. Jane Woyka. Consensus statement for non-hormonal-based treatments for menopausal symptoms. Post Reproductive Health. 2017; 23(2)71-75.

20. Antoine C, Liebens F, Carly B, et al. Safety of alternative treatments for menopausal symptoms after breast cancer: a qualitative systematic review. Climacteric. 2007;10(1):23-26.

21. Hutton B, Yazdi F, Bordeleau L, et al, Comparison of physical interventions, behavioral interventions, natural health products, and pharmacologics to manage hot flashes in patients with breast or prostate cancer: protocol for a systematic review incorporating network meta-analyses. Syst Rev. $2015 ; 4: 114$

22. Pandya KJ, Rauberta RF, Flyn PJ, et al. Oral clonidine in postmenopausa patients with breast cancer experiencing tamoxifen- induced hot flashes: a University of Rochester Cancer Center Community Clinical Oncology Program study. Ann Inter Med. 2000; 132(10):788-793.

23. Loibl S, Schwedler K, von Minckwitz G, et al. Venlafaxine is superior to clonidine as treatment of hot flashes in breast cancer patients - a doubleblind, randomized study. Ann Onc. 2007;18(4):689-693.

24. Nelson HD, Vesco KK, Haney E Rongwei F. Nonhormonal therapies for menopausal hot flashes: a systematic review and meta-analysis. JAMA 2006;295:2057-2071.

25. Stearns V. Clinical update: new treatments for hot flushes. Lancet. 2007;369(9579):2062-2064.
26. Speroff L, Gass M, Constantine G, et al. Efficacy and tolerability of desvenlafaxine succinate treatment for menopausal vasomotor symptoms: a randomized controlled trial. Obstet Gynecol. 2008;111(1):77-87.

27. Bardia A, Novotny P, Sloan J, et al. Efficacy of nonestrogenic hot flash therapies among women stratified by breast cancer history and tamoxifen use: a pooled analysis. Menopause. 2009;16(3):477-483.

28. Loprinzi C, Kugler J, Sloan J, et al. Venlafaxine in management of hot flashes in survivors of breast cancer: a randomized controlled trial. Lancet. 2000;356(9247):2059-2063.

29. Jin Y, Desta Z, Stearns V, et al. CYP2D6 genotype, antidepressant use, and tamoxifen metabolism during adjuvant breast cancer treatment. J Natl Cancer Inst. 2005;97(1):30-39.

30. Bourque F, Karama S, Looper K, et al. Acute Tamoxifen-Induced Depression and Its Prevention with Venlafaxine. Psychosomatics. 2009;50(2):162-165.

31. Carpenter J, Storniolo A, Johns S, et al. Randomized, Double-Blind, Placebo-Controlled Crossover Trials of Venlafaxine for Hot Flashes After Breast Cancer. The Oncologist. 2007;12(1):124-135.

32. Lugones L, Belardo A, Tutzer M. Venlafaxine for the management of hot flashes (Preliminary Work). IV Argentine journeys of Climacteric, Salta, Argentina; 2003

33. Schober C, Ansani N, Venlafaxine Hydrochloride for the Treatment of Ho Flashes. Ann Pharmacother. 2003;37:1703-1707.

34. Johnson ED, Carroll DG. Venlafaxine and desvenlafaxine in the management of menopausal hot flashes. Pharmacy Practice. 2011;9(3):117-121

35. Archer DF, Seidman L, Constantine GD, et al. A double-blind, randomly assigned, placebo-controlled study of desvenlafaxine efficacy and safety for the treatment of vasomotor symptoms associated with menopause. Am J Obstet Gynecol. 2009;200(2):172.e1-e10.

36. Thase M, Fayyad R, Cheng R, et al. Effects of desvenlafaxine on blood pressure in patients treated for major depressive disorder: a pooled analysis. Curr Med Res Opin. 2015;31(4):809-820.

37. Berhan Y, Berhan A. Is desvenlafaxine effective and safe in the treatment of menopausal vasomotor symptoms? A meta-analysis and metaregression of randomized double-blind controlled studies. Ethiop J Health Sci. 2014;24(3):209-216.

38. Hickey M, Saunders C, Partridge A, et al. Practical clinical guidelines for assessing and managing menopausal symptoms after breast cancer. Ann Oncol. 2008;19(10):1669-1680.

39. Mariani L, Quattrini M, Atlante M, et al. Hot-flashes in breast cancer survivors: effectiveness of low-dosage fluoxetine. A pilot study. J Exp Clin Cancer Res. 2005;24(3):373-378.

40. Suvanto-Luukkonen E, Koivunen R, Sundström H, et al. Citalopram and fluoxetine in the treatment of postmenopausal symptoms: a prospective, randomized, 9-month, placebo controlled, double-blind study. Menopause. 2005;12(1):18-26

41. Grady D, Cohen B, Tice J, et al. Ineffectiveness of sertraline for treatment of menopausal hot flushes: a randomized controlled trial. Obstet Gynecol. 2007;109(4):823-830.

42. Kerwin JP, Gordon PR, Senf JH, The variable response of women with menopausal hot flashes when treated with sertraline. Menopause. 2007;14(5):841-845.

43. Kimmick GG, Lovato J, McQuellon R, et al. Randomized, double-blind placebo-controlled, crossover study of sertraline (Zoloft) for the treatment 
of hot flashes in women with early stage breast cancer taking tamoxifen. Breast J. 2006;12(2):114-122.

44. Simon J, Portman D, Kaunitz A, et al. Low-dose paroxetine $7.5 \mathrm{mg}$ for menopausal VMS: two randomized controlled trials. Menopause. 2013;20(10):1027-35.

45. Capriglione S, Plotti F, Montera R, et al. Role of paroxetine in the management of hot flashes in gynecological cancer survivors: Results of the first randomized single-center controlled trial. Gynecologic Oncology. 143(2016):584-588.

46. Pinkerton J, Joffe H, Kazempour K, et al. Low-dose paroxetine (7.5 mg) improves sleep in women with vasomotor symptoms associated with menopause. Menopause. 2015;22(1):50-58.

47. Regan MM, Leyland-Jones B, Bouzyk M, et al. CYP2D6 genotype and tamoxifen response in postmenopausal women with endocrine-responsive breast cancer: the breast international group 1-98 trial. J Natl Cancer Inst. 2012;104(6):441-451.

48. Kelly CM, Juurlink DN, Gomes T, et al. Selective serotonin reuptake inhibitors and breast cancer mortality in women receiving tamoxifen: a population based cohort study. BMJ. 2010;8;340- c693.

49. Kostandinos S, Ingle J, Ames M, et al. Coprescription of Tamoxifen and Medications That Inhibit CYP2D6. J Clin Oncol. 2010;28(16):2768-2776.

50. Haque R, Shi J, Schottinger JE, et al. Tamoxifen and Antidepressant Drug Interaction in a Cohort of 16,887 Breast Cancer Survivors. J Natl Cancer Inst. 2015:1(3):108.

51. Kalay A, Demir B, Haberal A, et al. Efficacy of citalopram on climacteric symptoms. Menopause. 2007:14(2);223-229.

52. Loprinzi C, Flynn P, Carpenter L, et al. Pilot evaluation of citalopram for the treatment of hot flashes in women with inadequate benefit from venlafaxine. J Palliat Med. 2005;8(5):924-930.

53. Barton D, LaVasseur B, Sloan J, et al. Phase III placebo-controlled trial of three doses of citalopram for the treatment of hot flashes: NCCTG trial N05C9. J Clin Oncol. 2010;28(20):3278-3283.

54. Suvanto-Luukkonen E, Koivunen R, Sundström H, et al. Citalopram and fluoxetine in the treatment of postmenopausal symptoms: a prospective, randomized, 9-month, placebo-controlled, double blind study. Menopause. 2005;12(1):18-26.

55. DeFronzo Dobkin R, Menza M, Allen L, et al. Escitalopram reduces hot flashes in nondepressed menopausal women: A pilot study. Ann Clin Psychiatry. 2009;21(2):70-76.

56. Freeman E, Guthrie K, Caan B, Efficacy of Escitalopram for Hot Flashes in Healthy Menopausal Women A Randomized Controlled Trial. JAMA. 2011;305(3):267-274.
57. Belardo A, Starvaggi A, Bozza L. Evaluación de eficacia del escitalopram en el tratamiento de los sofocos en mujeres postmenopáusicas. Estudio piloto. Congreso AAPEC; 2014.

58. Guthrie K, LaCroix A, Ensrud K, et al. Pooled Analysis of Six Pharmacologic and Nonpharmacologic Interventions for Vasomotor Symptoms. Obstet Gynecol. 2015;126(2):413-422.

59. Loprinzi C, Barton D, Sloan J, et al, Mayo Clinic and North Central Cancer Treatment Group hot flash studies: a 20-year experience. Menopause. 2008;15(4 Pt 1):655-660.

60. Goetz MP, Suman V, Henry NL, et al, Tamoxifen in Women With Breast Cancer and in Women at High-Risk of Breast Cancer Who Are Receiving Venlafaxine, Citalopram, Escitalopram, Gabapentin, or Sertraline. results of a prospective multicenter study: (NCT 00667121), Mayo Clinic; 2008.

61. Pacheco L, Aragües M. Alternatives in the management of discontinuation syndrome with new antidepressants. Psiquiatr Biol. 2002;9:133-134.

62. Pandya K, Morrow G, Roscoe J, et al. Gabapentin for hot flashes in 420 women with breast cancer: a randomised double-blind placebo-controlled trial. The Lancet. 2005;366(9488):818-824.

63. Reddy SY, Warner H, Guttuso T, et al. Gabapentin, estrogen, and placebo for treating hot flushes: a randomized controlled trial. Obstet Gynecol. 2006;108 (1):41-48.

64. Butt DA, Lock M, Lewis J, et al. Gabapentin for the treatment of menopausal hot flashes: a randomized controlled trial. Menopause. 2008;15(2):310-318.

65. Bordelau L, Pritchard K, Loprinzi C, et al. Multicenter, randomized, crossover clinical trial for venlafaxine versus gabapentin for the management of hot flushes in breast cancer survivors. J Clin Oncol. 2010;28:5147-5152.

66. Rahmanian M, Mohseni A, Ghorbani R. A crossover study comparing gabapentin and fluoxetine for the treatment of vasomotor symptoms among postmenopausal women. Int J Gynaecol Obstet. 2015;131(1):8790 .

67. Agarwal N, Singh S, Kriplani A, et.al. Evaluation of gabapentin in management of hot flushes in postmenopausal women. Post Reprod Health. 2014;20(1):36-38.

68. Loprinzi CL, Qin R, Balcueva EP, et al. Phase III, randomized, doubleblind, placebo-controlled evaluation of pregabalin for alleviating hot flashes NO7C1. J Clin Oncol. 2010;28(4):641-647.

69. L'Espérance S, Frenette S, Dionne A, et al. Pharmacological and nonhormonal treatment of hot flashes in breast cancer survivors: CEPO review and recommendations. Support Care Cancer. 2013;21(5):1461-1474. 